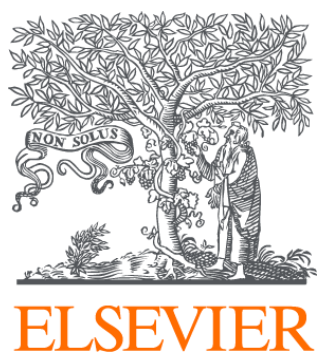

Since January 2020 Elsevier has created a COVID-19 resource centre with free information in English and Mandarin on the novel coronavirus COVID-

19. The COVID-19 resource centre is hosted on Elsevier Connect, the company's public news and information website.

Elsevier hereby grants permission to make all its COVID-19-related research that is available on the COVID-19 resource centre - including this research content - immediately available in PubMed Central and other publicly funded repositories, such as the WHO COVID database with rights for unrestricted research re-use and analyses in any form or by any means with acknowledgement of the original source. These permissions are granted for free by Elsevier for as long as the COVID-19 resource centre remains active. 


\title{
Respiratory rehabilitation in elderly patients with COVID-19: A randomized controlled study
}

\author{
Kai Liu ${ }^{\text {a,1 }}$, Weitong Zhang ${ }^{\mathrm{b}, 1}$, Yadong Yang ${ }^{\mathrm{c}, 1}$, Jinpeng Zhang ${ }^{\mathrm{c}, 1}$, Yunqian Li $^{\mathrm{a}}$, Ying Chen ${ }^{\mathrm{d},}$ \\ ${ }^{a}$ Department of Geriatric Center, Hainan General Hospital (Hainan Affiliated Hospital of Hainan Medical University), Haikou, 570311, PR China \\ ${ }^{\mathrm{b}}$ Department of General Surgery, Hainan General Hospital (Hainan Affiliated Hospital of Hainan Medical University), Haikou, 570311, PR China \\ ${ }^{\mathrm{c}}$ Department of Critical Care Medcine, Huanggang Central Hospital, Hubei Province. Huanggang, 438000, PR China \\ d Department of Medical Laboratory, Hainan General Hospital (Hainan Affiliated Hospital of Hainan Medical University), Haikou, 570311, China
}

\section{A R T I C L E I N F O}

\section{Keywords:}

Elderly

Covid-19

Respiratory rehabilitation

Pulmonary function

Quality of life

Psychological state

\begin{abstract}
A B S T R A C T
Background: Different degrees of disorders are reported in respiratory function, physical function and psychological function in patients with corona virus disease 2019 (COVID-19), especially in elderly patients. With the experience of improved and discharged COVID-19 patients, timely respiratory rehabilitation intervention may improve prognosis, maximize functional preservation and improve quality of life (QoL), but there lacks of studies worldwide exploring the outcome of this intervention.

Objective: To investigate the effects of 6-week respiratory rehabilitation training on respiratory function, QoL, mobility and psychological function in elderly patients with COVID-19.

Methods: This paper reported the findings of an observational, prospective, quasi-experimental study, which totally recruited 72 participants, of which 36 patients underwent respiratory rehabilitation and the rest without any rehabilitation intervention. The following outcomes were measured: pulmonary function tests including plethysmography and diffusing lung capacity for carbon monoxide (DLCO), functional tests (6-min walk distance test), Quality of life (QoL) assessments (SF-36 scores), activities of daily living (Functional Independence Measure, FIM scores), and mental status tests (SAS anxiety and SDS depression scores).

Results: After 6 weeks of respiratory rehabilitation in the intervention group, there disclosed significant differences in FEV1(L), FVC(L), FEV1/FVC\%, DLCO\% and 6-min walk test. The SF-36 scores, in 8 dimensions, were statistically significant within the intervention group and between the two groups. SAS and SDS scores in the intervention group decreased after the intervention, but only anxiety had significant statistical significance within and between the two groups.

Conclusions: Six-week respiratory rehabilitation can improve respiratory function, QoL and anxiety of elderly patients with COVID-19, but it has little significant improvement on depression in the elderly.
\end{abstract}

\section{Introduction}

2019-novel coronaviruses belong to the $\beta$ species of coronaviruses, which mainly transmitted through respiratory droplets and close contact, and it can be found in human respiratory epithelial cells in about $96 \mathrm{~h}$, which firstly attacks the lungs and induces serous fluid, fibrin exudates, and hyaline membrane formation in the alveoli [1]. COVID-19 infection is furiously spreading at an alarming rate, with a cumulative number of 116,736 confirmed cases and 5701 deaths outside China to date. As the earliest epidemic area, China has achieved a major victory in the cooperation of the people through the efforts of the government.
At present, a total of 70,547 cases have been cured and discharged in China. In this outbreak, the elderly population is generally susceptible with high incidence of severe disease and mortality [2]. Community-acquired pneumonia in the elderly population has been found to result in decreased activities of daily living (ADL) and QoL, accompanied by decreased physical and mental function [3]. Respiratory disorders and lack of exercise in the elderly can lead to diseases such as apraxia syndrome and pulmonary infections [4]. Therefore, for elderly patients who suffered from COVID-19 and discharged with satisfying results, improved respiratory function is an important factor in maintaining ADL and QoL of the elderly.

\footnotetext{
* Corresponding author.

E-mail address: hkchenying@126.com (Y. Chen).

1 Contributed equally.
} 
Respiratory rehabilitation can improve respiratory function and QoL in patients with chronic obstructive pulmonary disease (COPD) [5]. However, the effect of respiratory rehabilitation on respiratory function and QoL in older adults with COVID-19 is unknown. Studies have evaluated respiratory function in elderly patients with COPD, including those with suspected COPD, who have better respiratory rehabilitation [6]. Thus, in this study, we conducted a randomized controlled trial to investigate the effects of respiratory rehabilitation on respiratory function, ADL, QoL, and psychological status in elderly patients with COVID-19 who were discharged from the hospital with satisfying results.

\section{Method}

Participants: were recruited from Hainan General Hospital central hospital and Huanggang Central hospital, which were designated by the government to admit COVID-19 from January 1, 2020 to February 6, 2020. Inclusion Criteria: (1) with a definite diagnosis of COVID-19; (2) aged 65 years or above; (3) $\geq 6$ months after the onset of other acute diseases; (4) mini-mental state examination (MMSE) score $>21$; (6) no COPD or any other respiratory disease; and (7) forced expiratory volume in $1 \mathrm{~s}$ (FEV1) $\geq 70 \%$. Exclusion criteria: (1) moderate or severe heart disease (Grade III or IV, New York Heart Association); (2) with severe ischemic or hemorrhagic stroke or neurodegenerative diseases.

Study Design: This study is an open randomized controlled trial. Participants were aware of all rehabilitation procedures, including respiratory rehabilitation ( 2 sessions per week for 6 weeks), once a day for $10 \mathrm{~min}$. The demographic characteristics of each subject were assessed prior to randomizing the subject. Odd numbers of patients were in the intervention group while even numbers of patients in the control group using a computer-generated allocation order. Interventions included: (1) respiratory muscle training; (2) cough exercise; (3) diaphragmatic training; (4) stretching exercise; and (5) home exercise. For respiratory muscle training, participants used a commercial hand-held resistance device (Threshold PEP; Philips Co.) for three sets with 10 breaths in each set; parameters were set at $60 \%$ of the individual's maximal expiratory mouth pressure, with a rest period of $1 \mathrm{~min}$ between the two sets. Three sets of 10 active coughs were adopted for cough exercises. For diaphragmatic training, each participant performed 30 maximal voluntary diaphragmatic contractions in the supine position, placing a medium weight (1-3 kg) on the anterior abdominal wall to resist diaphragmatic descent. In stretching exercises, the respiratory muscles are stretched under the guidance of a rehabilitation therapist. The patient was placed in the supine or lateral decubitus position with the knees bent to correct the lumbar curve. Patients were ordered to move their arms in flexion, horizontal extension, abduction, and external rotation. In terms of home exercises, subjects were instructed in pursed-lip breathing and coughing training, and asked to undergo 30 sets per day.

\subsection{Assessment}

Primary Outcome Measures: Respiratory function; Secondary Outcome Measures: Exercise endurance (6-min walk distance), ADL and QoL, psychological status assessment (anxiety, depression scores).

Respiratory function, by automated computerized spirometer (Model ML3500S) of Micro Direct, for assessing respiratory function. The following parameters related to respiratory function were measured: (1) forced expiratory volume in 1 second (FEV1); (2) forced vital capacity (FVC); (3) DLCO (\%) refers to the amount of CO that passes through the alveolar capillary membrane into the capillary blood per unit time, per unit pressure difference with a percentage of the measured value to the predicted value $>80 \%$ as normal.

Exercise endurance, which measured under the 6-min walk test (6MWT), is the distance one walks within $6 \mathrm{~min}$ (also known as the dynamic distance). Percutaneous oxygen saturation $\left(\mathrm{SpO}_{2}\right)$, heart rate, systolic blood pressure, diastolic blood pressure, respiratory rate and perceived exertion (Borg scale) were measured before and after a 6-min walk using a saturated pulse oximeter.

Activities of daily living( $A D L)$ The rehabilitation therapist assessed ADL with the Functional Independence Measure (FIM) scale. The FIM contains 18 items, each with a maximum score of 7 points, minimum score of 1 point, and a maximum total score of 126 points. The 18 items of the FIM can be divided into 13 items for assessing motor ADL (including 6 self-care items, 2 sphincter control items, 3 transfer items, and 2 motor items) and 5 items for assessing cognitive ADL (including 2 items) for communication and 3 items for social cognition).

QoL Assessment The QoL of patients in the two groups before and after nursing intervention was assessed using the Short Form-36 (SF-36). The SF-36 scale had 8 dimensions, and each dimension was converted into a percentage score. The higher score, the better QoL.

Assessment of Anxiety, Depression Self-rating depression scale (SDS) and self-rating anxiety scale (SAS) were exploited to assess depression and anxiety in the two groups 2 days after the intervention. Both SDS and SAS have 20 items, each of which was scored on a scale of $1-4$, and the higher score, the more severe the degree of depression and anxiety.

Study Termination The study was terminated when any of the following was reached: worsening of subjective symptoms (dyspnea); $\mathrm{SpO}_{2}$ decreased by $85 \%$ or less; the heart rate increased to $85 \%$ or more of the predicted maximum heart rate.

\subsection{Statistical analysis}

SPSS 24.0 was used for all statistical analysis. The minimum sample size of each group was 26 , power was $80 \%$, and an $\alpha$ error was $5 \%$. The magnitudes of the effects were calculated using the Mann Whitney $U$ test with Cohen'd coefficient set to 0.8 . Therefore, the sample size of this study $(\mathrm{n}=72)$ has sufficient detection ability. To ensure a balanced random distribution, Fisher's exact test was used for gender differences between the intervention group and the control group at baseline, and unpaired $t$-test was used for age and other variable evaluation. Wilcoxon rank sum test was used to compare the results of each group with the baseline indicators. Using Mann Whitney $U$ test to compare the difference between the two groups, the statistical significance was set as $\mathrm{P}<$ 0.05 .

Ethical considerations The survey was conducted with the approval of the ethics committees of Hainan General Hospital and Huanggang Central Hospital (approval numbers: 19758 and 20200125). All participants provided written informed consent after receiving a complete written description of the trial.

\section{Results}

A total of 92 patients were assessed for eligibility. Among them, 9 patients disagreed with this study, 3 patients with FEV1 $\leq 70 \%, 4$ patients severe heart disease, and the remaining 76 patients were randomized. Of the 38 patients in the intervention group, 2 patients abandoned before completing all 12 sessions and were unable to continue rehabilitation. Of the 38 patients in the control group, 2 were also unable to continue rehabilitation. Finally, a total of 72 patients completed the study, of whom 36 completed the respiratory rehabilitation program.

Baseline Characteristics There were no statistically significant differences between the two groups of patients in age, gender, BMI, extent of lung CT lesions, past medical history (Table 1).

Pulmonary Function Test The intervention group and the control group were compared after 6 weeks of respiratory rehabilitation, and found that there was a statistically significant difference between FEV1 (L), FVC (L), FEV1/FVC\% and DLCO\% (Fig. 1, Fig. 2, Table 2).

Exercise Capacity Test The 6-min walk distance after 6 weeks of respiratory rehabilitation within the intervention group was significantly longer than that before the intervention, which was statistically 
Table 1

Baseline characteristics for COVID-19 patients: Intervention versus Control group.

\begin{tabular}{|c|c|c|c|}
\hline Characteristics & $\begin{array}{l}\text { Intervention group ( } \mathrm{n}= \\
36 \text { ) }\end{array}$ & $\begin{array}{l}\text { Control group }(\mathrm{n}= \\
\text { 36) }\end{array}$ & $\begin{array}{l}\mathrm{P} \text { - } \\
\text { values }\end{array}$ \\
\hline Male, n, \% & $24(66.7)$ & $25(69.4)$ & 0.17 \\
\hline Age, years, $(\mathrm{M} \pm \mathrm{SD})$ & $69.4(8.0)$ & $68.9(7.6)$ & 0.24 \\
\hline $\begin{array}{l}\text { BMI, } \mathrm{kg} / \mathrm{m}^{2}, \\
\quad(\mathrm{M} \pm \mathrm{SD})\end{array}$ & $23.1(3.5)$ & $22.9(3.9)$ & 0.12 \\
\hline \multicolumn{4}{|c|}{ CT features of lung lesions, $\mathrm{n}$} \\
\hline Multilobular lesion & $25(69.4)$ & $23(63.9)$ & 0.33 \\
\hline Unilobar lesion & $11(30.6)$ & $13(36.1)$ & 0.27 \\
\hline Pleural effusion & $4(11.1)$ & $3(8.3)$ & 0.18 \\
\hline \multicolumn{4}{|l|}{ Comorbidity, n } \\
\hline Hypertension & $10(27.8)$ & $8(22.2)$ & 0.56 \\
\hline $\mathrm{T}_{2} \mathrm{DM}$ & $9(25.0)$ & $9(25.0)$ & 0.67 \\
\hline Osteoporosis & $8(22.2)$ & $6(16.7)$ & 0.41 \\
\hline
\end{tabular}

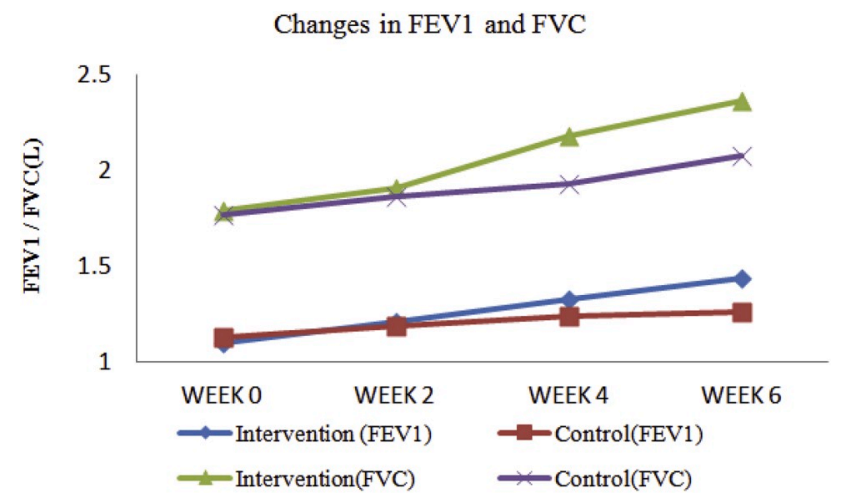

Fig. 1. Changes in FEV1 and FVC over a 6 week time frame for the full cohort of patients.

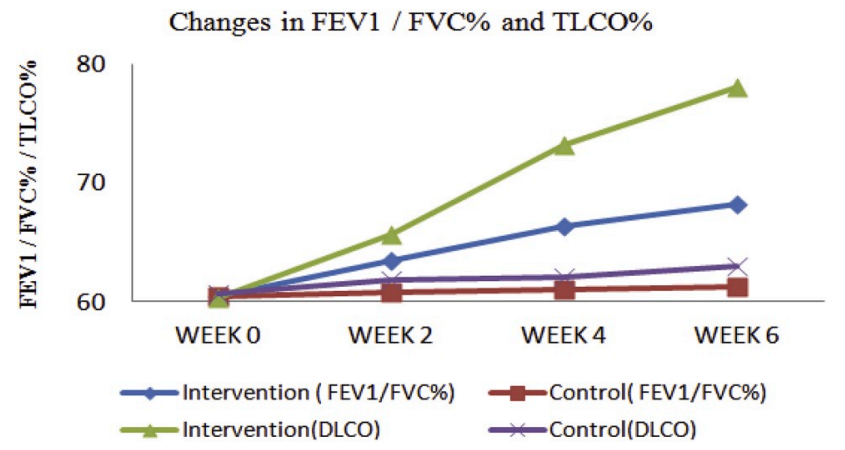

Fig. 2. Changes in FEV1/FVC\% and TLCO\% over a 6 week time frame for the full cohort of patients.

significant and also statistically significant compared with the control group (Fig. 3, Table 2).

ADL There was no significant improvement neither within the intervention group nor compared with the control group (Table 2).

QoL SF-36 scores in 8 dimensions, which were statistically significant within the intervention group and between the two groups, suggesting an improvement in QoL (Table 2).

Anxiety and Depression Scores SAS and SDS scores decreased after the intervention in the intervention group, but only anxiety was statistically significant within and between groups, and SDS scores were not statistically significant within and between groups (Table 2).

\section{Discussion}

To our knowledge, this first randomized controlled trial of COVID-19
Table 2

Comparison of lung function, quality of life, and anxiety and depression between the two groups before and after intervention $(M \pm S D)$.

\begin{tabular}{|c|c|c|c|c|}
\hline \multirow[b]{2}{*}{ Measures } & \multicolumn{2}{|c|}{ Intervention group $(\mathrm{n}=36)$} & \multicolumn{2}{|c|}{ Control group $(\mathrm{n}=36)$} \\
\hline & Pre & Post & $\begin{array}{l}6 \text { weeks } \\
\text { ago }\end{array}$ & $\begin{array}{l}\text { After } 6 \\
\text { weeks }\end{array}$ \\
\hline \multicolumn{5}{|c|}{ Pulmonary Function Test } \\
\hline FEV1(L) & $\begin{array}{l}1.10 \pm \\
0.08\end{array}$ & $\begin{array}{l}1.44 \pm \\
0.25^{\star \#}\end{array}$ & $1.13 \pm 0.14$ & $1.26 \pm 0.32$ \\
\hline FVC(L) & $\begin{array}{l}1.79 \pm \\
0.53\end{array}$ & $\begin{array}{l}2.36 \pm \\
0.49 * \#\end{array}$ & $1.77 \pm 0.64$ & $2.08 \pm 0.37$ \\
\hline FEV1/FVC\% & $\begin{array}{l}60.48 \pm \\
6.39\end{array}$ & $\begin{array}{l}68.19 \pm \\
6.05^{* \#}\end{array}$ & $\begin{array}{l}60.44 \pm \\
5.77\end{array}$ & $\begin{array}{l}61.23 \pm \\
6.43\end{array}$ \\
\hline TLCO \% & $\begin{array}{l}60.3 \pm \\
11.3\end{array}$ & $\begin{array}{l}78.1 \pm \\
12.3^{* \#}\end{array}$ & $60.7 \pm 12.0$ & $63.0 \pm 13.4$ \\
\hline \multicolumn{5}{|c|}{ Exercise Capacity Test } \\
\hline 6MWT, m & $\begin{array}{l}162.7 \pm \\
72.0\end{array}$ & $\begin{array}{l}212.3 \pm \\
82.5^{* \#}\end{array}$ & $\begin{array}{l}155.7 \pm \\
82.1\end{array}$ & $\begin{array}{l}157.2 \pm \\
71.7\end{array}$ \\
\hline \multicolumn{5}{|l|}{$A D L$} \\
\hline FIM & $109.2 \pm 13$ & $109.4 \pm 11.1$ & $\begin{array}{l}109.3 \pm \\
10.7\end{array}$ & $\begin{array}{l}108.9 \pm \\
10.1\end{array}$ \\
\hline \multicolumn{5}{|l|}{ QoL (SF-36) } \\
\hline Physical health & $52.4 \pm 6.2$ & $71.6 \pm 7.6^{* \#}$ & $53.2 \pm 7.7$ & $54.1 \pm 7.5$ \\
\hline Body role function & $61.2 \pm 6.6$ & $75.9 \pm 7.9^{* \#}$ & $61.3 \pm 7.2$ & $62.0 \pm 7.3$ \\
\hline Physical pain & $63.5 \pm 7.4$ & $78.3 \pm 7.8^{* \#}$ & $63.5 \pm 8.1$ & $62.9 \pm 7.9$ \\
\hline General health & $61.8 \pm 7.7$ & $74.2 \pm 7.9^{* \#}$ & $61.8 \pm 8.4$ & $61.4 \pm 6.9$ \\
\hline Energy & $60.6 \pm 6.9$ & $75.6 \pm 7.1^{* \#}$ & $60.5 \pm 7.1$ & $61.2 \pm 6.3$ \\
\hline Social function & $59.4 \pm 7.2$ & $69.8 \pm 6.4^{* \#}$ & $59.5 \pm 7.0$ & $58.9 \pm 6.6$ \\
\hline $\begin{array}{l}\text { Emotional role } \\
\text { function }\end{array}$ & $61.4 \pm 6.9$ & $75.7 \pm 7.0^{* \#}$ & $61.4 \pm 7.3$ & $60.8 \pm 7.3$ \\
\hline Mental health & $61.5 \pm 6.5$ & $73.7 \pm 7.6^{* \#}$ & $61.6 \pm 7.2$ & $62.1 \pm 7.6$ \\
\hline \multicolumn{5}{|c|}{ Anxiety and depression assessment } \\
\hline SAS score & $56.3 \pm 8.1$ & $47.4 \pm 6.3^{\star \#}$ & $55.8 \pm 7.4$ & $54.9 \pm 7.3$ \\
\hline SDS score & $56.4 \pm 7.9$ & $54.5 \pm 5.9$ & $55.9 \pm 7.3$ & $55.8 \pm 7.1$ \\
\hline
\end{tabular}

* Compared with the same group after intervention, $\mathrm{P}<0.05$.

${ }^{\#}$ Compared with the control group after intervention, $\mathrm{P}<0.05$.

FVC: forced vital capacity; FEV1: forced expiratory volume at $1 \mathrm{~s}$; DLCO: diffusing lung capacity for carbon monoxide; 6MWT: 6-Minute Walk Test; FIM: Functional Independence Measure.

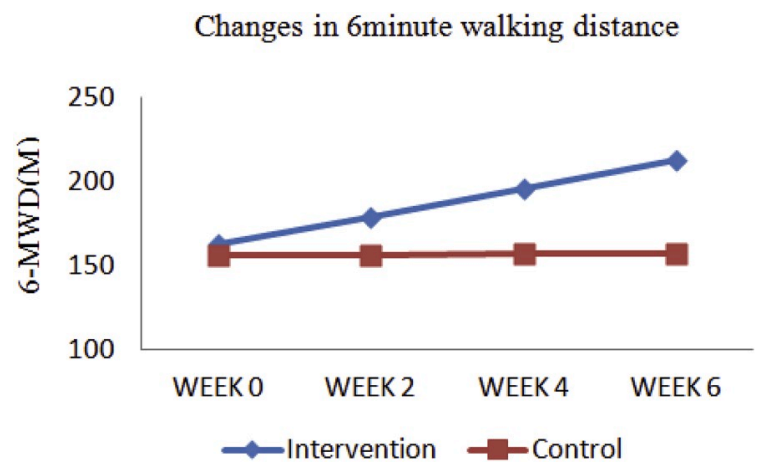

Fig. 3. Changes in 6 min walking distance over a 6 week time frame for the full cohort of patients.

in patients aims at investigating the efficacy of this regimen and revealing that 6-week respiratory rehabilitation significantly improves respiratory function, QoL, and anxiety and depression in elderly patients with COVID-19 and those without COPD.

Under CT, patients with COVID-19 may have some residual fibrotic lesions in the lungs following current treatment and discharge protocols [7], which may affect the patient's respiratory function. However, our study found that pulmonary function was significantly improved after 6 weeks of respiratory rehabilitation training. The reason may be that the rehabilitation training related to respiratory muscles in respiratory rehabilitation training, and respiratory muscles include intercostal muscles, enthusiastic muscles, abdominal wall muscles, etc., which play an important role in maintaining respiratory function. The decline of its 
function leads to dyspnea, abdominal breathing with labial constriction, increases the expansion range of the breast muscle during breathing, encourages patients to exercise the abdominal wall during breathing to reduce chest wall movement, slows down the respiratory rate to reduce power consumption, and increases pulmonary ventilation and blood oxygen content [8].

Our exercise endurance measures assessed using the 6-min walk test led to significant improvements in exercise capacity for the intervention after a 6-week respiratory rehabilitation program. These results are similar to those reported by Giansanti [9], who reported a significant improvement in 6MWD after 6-9 weeks of respiratory rehabilitation, suggesting an improvement in exercise capacity. However, exercise training is the core of respiratory rehabilitation, its effect is affected by the way, intensity, time and place of exercise training, and reasonable exercise training has a positive impact on the physical and mental health and QoL of COVID-19 patients [10]. Maki [11] et al. evaluated a study of 2504 patients with chronic obstructive non-disease who received exercise intervention and found that the patients' muscle strength increased by $78 \%$, muscle endurance increased by $92 \%$ and muscle mass increased by $88 \%$. The mechanism of action of exercise training on COPD rehabilitation is mostly related to the improvement of ventilation and gas exchange function, cardiovascular function and limb muscle function in patients [12]. Combined with our data, it is suggested that exercise training has a significant improvement on exercise capacity in COVID-19 patients.

In our study, living ability did not improve significantly after respiratory rehabilitation, which may be due to the relatively short duration of our respiratory rehabilitation. In addition, the independence of living ability of our patients was weak, so the improvement of living ability was not significant after short-term respiratory rehabilitation. In our study, QoL was assessed using the SF-36, and there was an improvement in QoL after 6 weeks of respiratory rehabilitation, which was statistically significant with the control group. Studies have found that: respiratory rehabilitation can improve the QoL of patients with COPD [6], asthma [13], lung cancer after surgery [14]. Additionally, we found that 6 weeks of respiratory rehabilitation significantly improved anxiety in elderly patients with COVID-19, which is consistent with the effect of respiratory rehabilitation in COPD with Rebelo [15]. While in our previous study, it has been found that patients with COVID-19 experience increased depression and anxiety after isolation treatment [16]. On the other hand, positive changes in depression scores did not seem to be influenced after the respiratory rehabilitation program. This is in line with the findings of McNamara [17] et al. who reported that 6-9 weeks of respiratory rehabilitation did not improve depression in elderly patients with COPD. The above results need to be further confirmed by samples and longer respiratory rehabilitation studies.

Conclusions: Six-week respiratory rehabilitation can improve respiratory function, QoL and anxiety in elderly patients with COVID-19, but it has no significant improvement in elderly depressive state and activities of daily living.

\subsection{Study limitations}

This study has several limitations. Owing to the nature of the recovery and assessment environment, every effort has been made to blind assessors and participants to group allocation, but this cannot be guaranteed. Therefore, we cannot rule out placebo effects, observer bias or experimenter bias in the current study. In addition, because at least 6 weeks of rehabilitation were required, we could only discharge patients before February 6, which make our the sample size small; to address these limitations, a further double-blind study with a large sample size at multiple centers was required.

\section{Contributors}

WTZ, YDY, JPZ, Respiratory rehabilitation guidance and data collection. KL and YC drafted the manuscript. YQL and KL revised the final draft. YC. KL was the main supervisor and initiator of this study and responsible for summarizing all data.

\section{References}

[1] Y.C. Wu, C.S. Chen, Y.J. Chan, The outbreak of COVID-19: an overview, J. Chin. Med. Assoc. 83 (2020) 217-220, https://doi.org/10.1097/ JCMA.0000000000000270.

[2] M.A. Johansson, D. Saderi, Open peer-review platform for COVID-19 preprints, Nature 579 (2020) 29, https://doi.org/10.1038/d41586-020-00613-4.

[3] A. Mikolajewska, M. Witzenrath, Community-acquired pneumonia in adults, Dtsch. Med. Wochenschr. 145 (2020) 359-370, https://doi.org/10.1055/a-0993-0874.

[4] T. Jo, H. Yasunaga, N. Michihata, et al., Influence of Parkinsonism on outcomes of elderly pneumonia patients, Park. Relat. Disord. 54 (2018) 25-29, https://doi.org/ 10.1016/j.parkreldis.2018.03.028.

[5] N. Maki, H. Sakamoto, Y. Takata, et al., Effect of respiratory rehabilitation for frail older patients with musculoskeletal disorders: a randomized controlled trial, J. Rehabil. Med. 50 (2018) 908-913, https://doi.org/10.2340/16501977-2490.

[6] M.J. Prunera-Pardell, S. Padín-López, A. Domenech-Del Rio, et al., Effectiveness of a respiratory rehabilitation programme in patients with chronic obstructive pulmonary disease, Enferm Clin. 28 (2018) 5-12, https://doi.org/10.1016/j. enfcli.2017.11.001.

[7] A.J. Rodriguez-Morales, J.A. Cardona-Ospina, E. Gutiérrez-Ocampo, et al., Clinical, laboratory and imaging features of COVID-19: a systematic review and metaanalysis, Travel Med. Infect. Dis. (2020) 101623, https://doi.org/10.1016/j. tmaid.2020.101623.

[8] L. Cui, H. Liu, L. Sun, Multidisciplinary respiratory rehabilitation in combination with non-invasive positive pressure ventilation in the treatment of elderly patients with severe chronic obstructive pulmonary disease, Pak. J. Med. Sci. 35 (2019) 500-505, https://doi.org/10.12669/pjms.35.2.459.

[9] D. Giansanti, G. Maccioni, Toward the integration of devices for pulmonary respiratory rehabilitation in telemedicine and e-health, Telemed. J. e Health 25 (3) (2019) 257-259, https://doi.org/10.1089/tmj.2018.0057.

[10] Chinese Association of Rehabilitation Medicine, Recommendations for respiratory rehabilitation of COVID-19 in adult, Zhonghua Jiehe He Huxi Zazhi 43 (2020) E029, https://doi.org/10.3760/cma.j.cn112147-20200228-00206, 0.

[11] N. Maki, H. Sakamoto, Y. Takata, et al., Effect of respiratory rehabilitation for frail older patients with musculoskeletal disorders: a randomized controlled trial, J. Rehabil. Med. 50 (10) (2018) 908-913, https://doi.org/10.2340/165019772490 .

[12] J. Levy, H. Prigent, D. Bensmail, Respiratory rehabilitation in multiple sclerosis: a narrative review of rehabilitation techniques, Ann. Phys. Rehabil. Med. 61 (1) (2018 Jan) 38-45, https://doi.org/10.1016/j.rehab.2017.06.002.

[13] T. Schneeberger, I. Jarosch, J. Moll, et al., Ncreased asthma control after a 3-week inpatient pulmonary rehabilitation program, Respir. Med. 165 (2020 Mar 8) 105930, https://doi.org/10.1016/j.rmed.2020.105930.

[14] K. Wytrychowski, A. Hans-Wytrychowska, P. Piesiak, et al., Pulmonary rehabilitation in interstitial lung diseases: a review of the literature, Adv. Clin. Exp. Med. 29 (2) (2020 Feb) 257-264, https://doi.org/10.17219/acem/115238.

[15] P. Rebelo, A. Oliveira, L. Andrade, et al., Minimal clinically important differences for patient-reported outcome measures of fatigue in patients with COPD after pulmonary rehabilitation, Chest (20) (2020 Mar 14) 30442-30446, https://doi. org/10.1016/j.chest.2020.02.045, pii: S0012-3692.

[16] Kai Liu, Ying Chen, Duozhi Wu, et al., Effects of progressive muscle relaxation on anxiety and sleep quality in patients with COVID-19, Compl. Ther. Clin. Pract. 39 (2020) 101132, https://doi.org/10.1016/j.ctcp.2020.101132.

[17] R.J. McNamara, M. Dale, Z.J. McKeough, Innovative strategies to improve the reach and engagement in pulmonary rehabilitation, J. Thorac. Dis. 11 (Suppl 17) (2019 Oct) S2192-S2199, https://doi.org/10.21037/jtd.2019.10.29. 